\title{
NEURODEGENERATIVE DISEASE
}

\section{Screening Zebrafish for Parkinson's disease drugs}

Kim, G-H. J. et al. eLife 10, e69795 (2021)

Parkinson's disease (PD) is a common neurodegenerative disorder caused by the loss of dopamine (DA)-producing neurons in the substantia nigra of the midbrain. Current pharmacological treatments, focusing on DA replacement, only provide symptomatic relief and are associated with adverse effects. Efforts are therefore underway to discover new compounds that can effectively and safely slow the progression of PD.

In eLife, Kim and colleagues used a zebrafish model of PD to screen $>1,400$ bioactive molecules for neuroprotective properties. The high-throughput phenotypic screen combined the use of a chemogenetic DA-neuron ablation zebrafish model - a model in which addition of metronidazole (MTZ) to zebrafish expressing nitroreductase (NTR) in DA neurons results in robust DA neuron loss - and in vivo brain imaging to assess DA neuron integrity in the fish. The chemical screen identified several agents that protected against DA neuron loss, including compounds targeting the Renin-Angiotensin-Aldosterone System (RAAS).

Previous studies have reported neuroprotective effects for RAAS inhibitors, but the underlying mechanisms were not well characterized. By showing that RAAS inhibitors could prevent DA neuron loss in a zebrafish model of PD in which neuronal death is preceded by mitochondrial DNA damage and subsequent mitochondrial dysfunction, the new findings suggest that RAAS inhibitors exert their neuroprotective action by restoring mitochondrial function.

The study also shows that RAAS inhibitors protected DA neurons in two other zebrafish models of neurodegeneration (a chemically induced Gaucher disease model and an MPP+ model) and in a pink1-deficient Drosophila model of PD, demonstrating that the protective effects of the RAAS inhibitors are conserved across models of neurodegeneration and species.

The RAAS pathway is classically known for regulating vasoconstriction and water homeostasis, and RAAS inhibitors are cornerstones in the treatment of several cardiovascular diseases, including hypertension. According to these results, repurposing RAAS inhibitors for the treatment of brain disorders could be of immediate and major translational value. "RAAS pathway components are broadly expressed in the CNS, suggesting that its inhibition could be broadly neuroprotective, not only for PD, but also for other neurodegenerative diseases," conclude the researchers in their report.

Alexandra Le Bras

Published online: 6 October 2021

https://doi.org/10.1038/s41684-021-00878-w

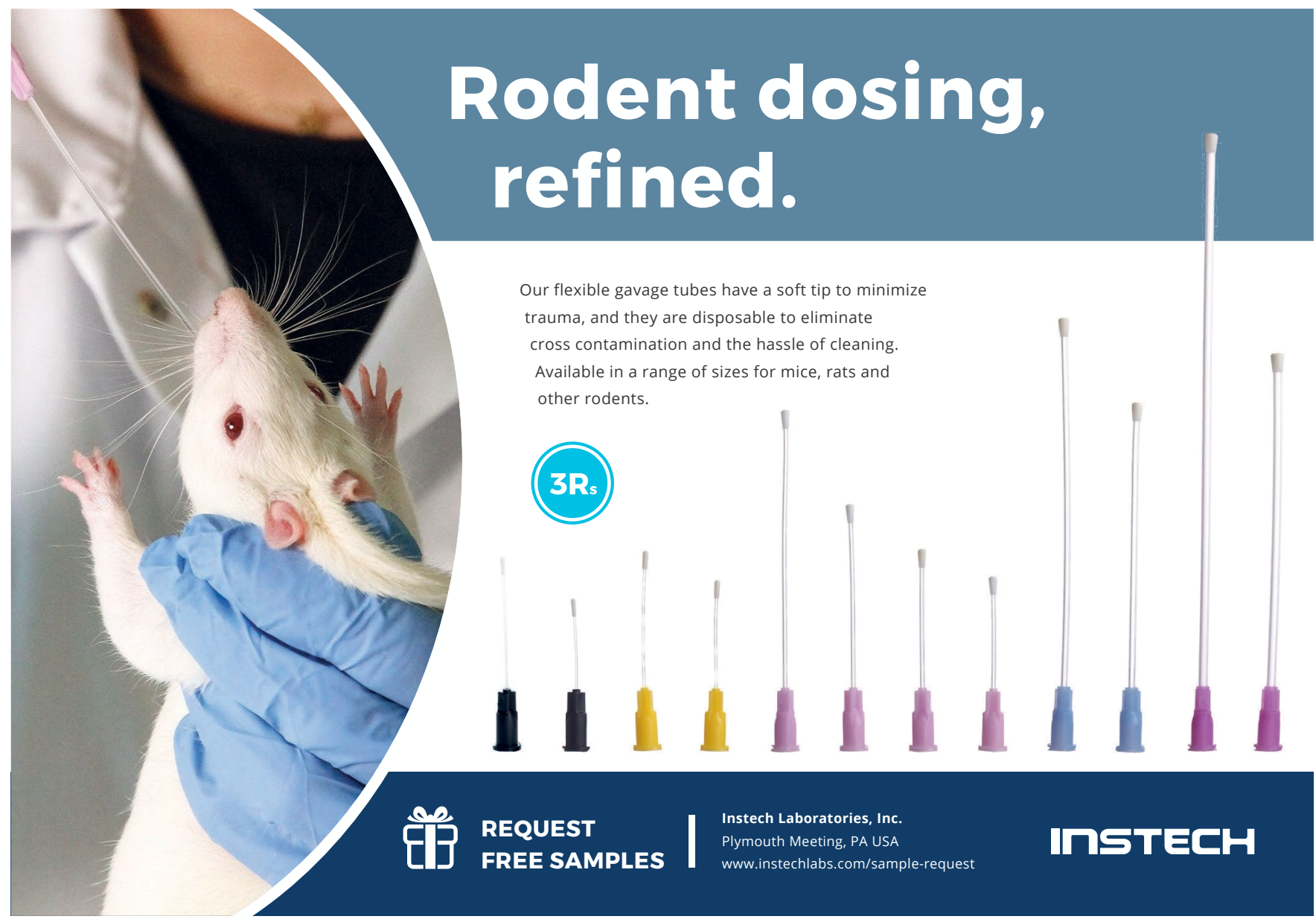

